\title{
Enhanced cellular responses and environmental sampling within inner foreskin explants: implications for the foreskin's role in HIV transmission
}

\author{
KM Fahrbach ${ }^{1}$, SM Barry ${ }^{1}$, MR Anderson ${ }^{1}$ and TJ Hope ${ }^{1}$
}

The decrease in HIV acquisition after circumcision suggests a role for the foreskin in HIV transmission. However, the mechanism leading to protection remains undefined. Using tissue explant cultures we found that Langerhans cells (LCs) in foreskin alter their cellular protein expression in response to external stimuli. Furthermore, we observe that upon treatment with TNF- $\alpha$, tissue-resident LCs became activated and that stimulatory cytokines can specifically cause an influx of CD4 + T-cells into the epithelial layer. Importantly, both of these changes are significant in the inner, but not outer, foreskin. In addition, we find that LCs in the inner foreskin have increased ability to sample environmental proteins. These results suggest differences in permeability between the inner and outer foreskin and indicate that HIV target cells in the inner foreskin have increased interaction with external factors. This increased responsiveness and sampling provides novel insights into the underlying mechanism of how circumcision can decrease HIV transmission.

\section{INTRODUCTION}

Sexual transmission of HIV requires that virions interact with HIV target cells in the genital epithelium within and beneath protective barriers. For example, the close proximity of Langerhans cells (LCs) to the epithelial surface and their ability to extend processes make them likely to be the first cells to encounter invading pathogens. ${ }^{1-3}$ The primary target of HIV, $\mathrm{CD} 4+\mathrm{T}$-cells, typically reside below the squamous epithelium and are less likely to be exposed to external agents. However, during inflammation, CD4 + T-cells can infiltrate into the superficial epithelium. How HIV reaches and infects these or other target cells remains to be elucidated.

Clues to the mechanism of sexual transmission may come from circumstances that increase its efficiency, such as inflammation. For example, pre-existing sexually transmitted infections cause an influx of immune cells to the genital tissue. Susceptibility to HIV infection (and multivariate HIV infection) is increased when other sexually transmitted infections are already established. ${ }^{4-8}$ Therefore, in the course of sexually transmitted infections, immune cells can migrate into the epithelium and/or become activated and facilitate infection by HIV virions. The presence of the foreskin also has been shown to increase the efficiency of transmission as multiple studies have shown that circumcision decreases the rate of female-to-male transmission by more than $50 \% .^{9-13}$

The effect of the foreskin on the efficiency of HIV transmission is currently open to debate. Recent reports observe no major differences in keratin thickness, target cell density, or location between the outer or inner foreskin, or glans tissue. ${ }^{14}$ Therefore, other physical characteristics or cellular responses may account for the increased rate of female-to-male transmission associated with the presence of the foreskin.

To gain insights into the possible mechanism, we used foreskin tissue explants to study the localization and activation state of resident HIV target cells. We found that the LCs sustain dynamic regulation of their surface markers, including a marker of activation. An infiltration of CD4 + T-cells was also observed after treatment of inner foreskin explants with specific inflammatory cytokines. In addition, there was a greater ability of LCs in the inner foreskin, over those in the outer foreskin, to sample external antigen. Together, these experiments show an enhanced ability of the immune cells of the inner foreskin to

${ }^{1}$ Department of Cell and Molecular Biology, Feinberg School of Medicine, Northwestern University, Chicago, Illinois, USA. Correspondence: TJ Hope (tjhope@northwestern.edu).

Received 25 November 2009; accepted 20 March 2010; published online 21 April 2010. doi:10.1038/mi.2010.18 
interact with and respond to environmental stimuli. We propose that these interactions result in increased local inflammation. Subsequently, recruitment and activation of resident target cells is in part responsible for the increased efficiency of HIV acquisition associated with the presence of the foreskin.

\section{RESULTS}

\section{Modulation of LC marker expression by DNFB}

We were interested in studying immune cells within the foreskin epithelium and determine whether we could modify their behavior with specific treatments. As LCs reside near the epithelial surface and have projections that can sample proteins in the environment, we began by trying to manipulate their activity. Takashima and co-workers ${ }^{15}$ observed an increase in the movement of LC projections after exposing human LCs transplanted into mouse ears to the skin irritant dinitrofluorobenzene (DNFB). Dynamic movement of LCs could potentially increase their interactions with and capture of invading pathogens. In addition, loss of LCs has been observed in epithelial tissue after exposure to DNFB. ${ }^{16}$ To determine the effect of DNFB on LCs in foreskin, explants of outer and inner foreskin were either cultured in media alone or exposed to $0.2 \%$ DNFB for periods of 4 to $6 \mathrm{~h}$. After explants were frozen and sectioned, they were stained for CD1a, a protein highly expressed on LCs and commonly used as an LC marker. ${ }^{17}$ A robust and clear CD1a signal identifies each of the LCs (green haze of stain in the stratum corneum is an artifact of staining with our CD1a antibody after fixation and does not represent true cellular signal). Sections were also stained with Hoechst and wheat-germ agglutinin (WGA), which stains nuclei and cell-surface polysaccharides, respectively, to assure the tissue had maintained its structural integrity. We immediately observed a decrease in CD1a-positive cells in both outer and inner foreskin after DNFB exposure (Figure 1a). We hypothesized that DNFB exposure had stimulated the LCs to emigrate from the epithelium. To confirm the actual loss of LCs from the tissue, sections from the same explants were co-stained with CD1a and the LC-specific marker langerin. ${ }^{18}$ Langerin-positive cells also stained positive for CD1a in the untreated explants. Surprisingly, we were able to identify langerin-positive, CD1a-negative cells in the DNFBexposed explants of both outer (Figure 1a) and inner foreskin (Supplementary Figure 1 online). Data were quantified by first calculating the number of CDla-positive cells $/ 100 \mu \mathrm{m}^{2}$ of epithelium in untreated explants. When these explants were co-stained with anti-CD1a and anti-langerin antibodies we observed that all langerin-positive cells were also CD1a-positive. Therefore, staining with either marker in untreated tissue was considered $100 \%$ labeling, to which the number of CD1a- or langerin-positive cells in DNFB-treated samples was normalized (Figure 1b). Only $18.9 \% \pm 10.5$ of the CD1a-positive cells remained in the outer foreskin, and only $24.8 \% \pm 10.7$ were identified in the inner foreskin. The percent of langerin-positive cells in the DNFBtreated explants was also determined. Overall, there was less of a reduction in langerin-positive than CD1a-positive cells in both outer and inner foreskin explants treated with DNFB ( $46.1 \% \pm 12.3$ and $56.41 \% \pm 9.7$, respectively). This indicated that
LCs were in the tissue, but had downregulated their expression of CD1a after exposure to DNFB.

This regulation of CD1a expression shows a specific and robust response of LCs in the tissue explants. We had previously reported that the explant cultures remain intact over several days in culture. ${ }^{14}$ Therefore, we sought to determine whether CD1a expression could recover after DNFB removal. A new group of outer foreskin explants from those used in Figure 1 were treated with DNFB for 3-4h, were thoroughly washed with media, and then cultured for 2 days in the absence of DNFB. The percentage of CD1a-positive cells was calculated (as in Figure 1) in these washout explants (48wash) and was compared with those in $4 \mathrm{~h}$ untreated-and DNFB-exposed explants from the same specimen. Again, there was a decrease in CD1a-positive cells, to $18 \% \pm 8.6$, after a 4 -h exposure to DNFB (Figure 2). Importantly, explants that remained in culture an additional 2 days after DNFB removal contained $55 \% \pm 21.3$ CD1a-positive cells. Recovery of CD1a expression can be seen in the representative images in Figure 2. This indicates that LCs in foreskin explants maintain dynamic responsiveness to external stimuli over multiple days in culture.

\section{Activation of LCs by TNF- $\alpha$ in the inner foreskin}

DNFB had a very dramatic effect on the state of LCs in the genital epithelium and showed the multi-day responsiveness of the explant cultures. To extend these observations we next examined whether the resident LCs would respond to external inflammatory stimuli and if there were any differences in the response of the outer and inner foreskin. Our and other laboratories have reported the ability to activate either emigrant or derived LCs with tumor necrosis factor- $\alpha$ (TNF- $\alpha$ ) in vitro. ${ }^{19,20}$ Therefore, we exposed foreskin explants to TNF- $\alpha$ for a period of 2-3 days before analysis. The epithelial area of each image was determined and the number of LCs was counted to determine an average number of $\mathrm{LCs} / 100 \mu \mathrm{m}^{2}$ of epithelium from multiple specimens. Upon comparing untreated and treated explants, we observed minimal, if any, physiological differences in LC size or localization within the epithelium (data not shown). With TNF- $\alpha$, there were nominal increases of 1.4- and 1.5-fold in the cell number in the inner and outer foreskin, respectively (Figure 3).

A substantial increase in cell number is typically indicative of an inflammatory response; however, as the vast majority of LCs naturally reside in the epithelium, it was not surprising to find a minimal increase in total cell number upon stimulation. Therefore, we expanded our analysis to include staining for activation marker CD86 in conjunction with CD1a. CD86 is a costimulatory molecule upregulated on dendritic cells after their activation. ${ }^{21}$ Using the TNF- $\alpha$-treated explants as described above, frozen sections were stained for both CD1a and CD86, blinded, and analyzed to identify activated LCs. Figure 4a shows examples of increased CD86 expression on LCs in inner foreskin explants treated with TNF- $\alpha$. In this sample image there are four LCs with visibly upregulated CD86 (red, second column) with TNF- $\alpha$ (bottom row); the insets show an enlargement of one of these cells. This is compared with untreated explants 

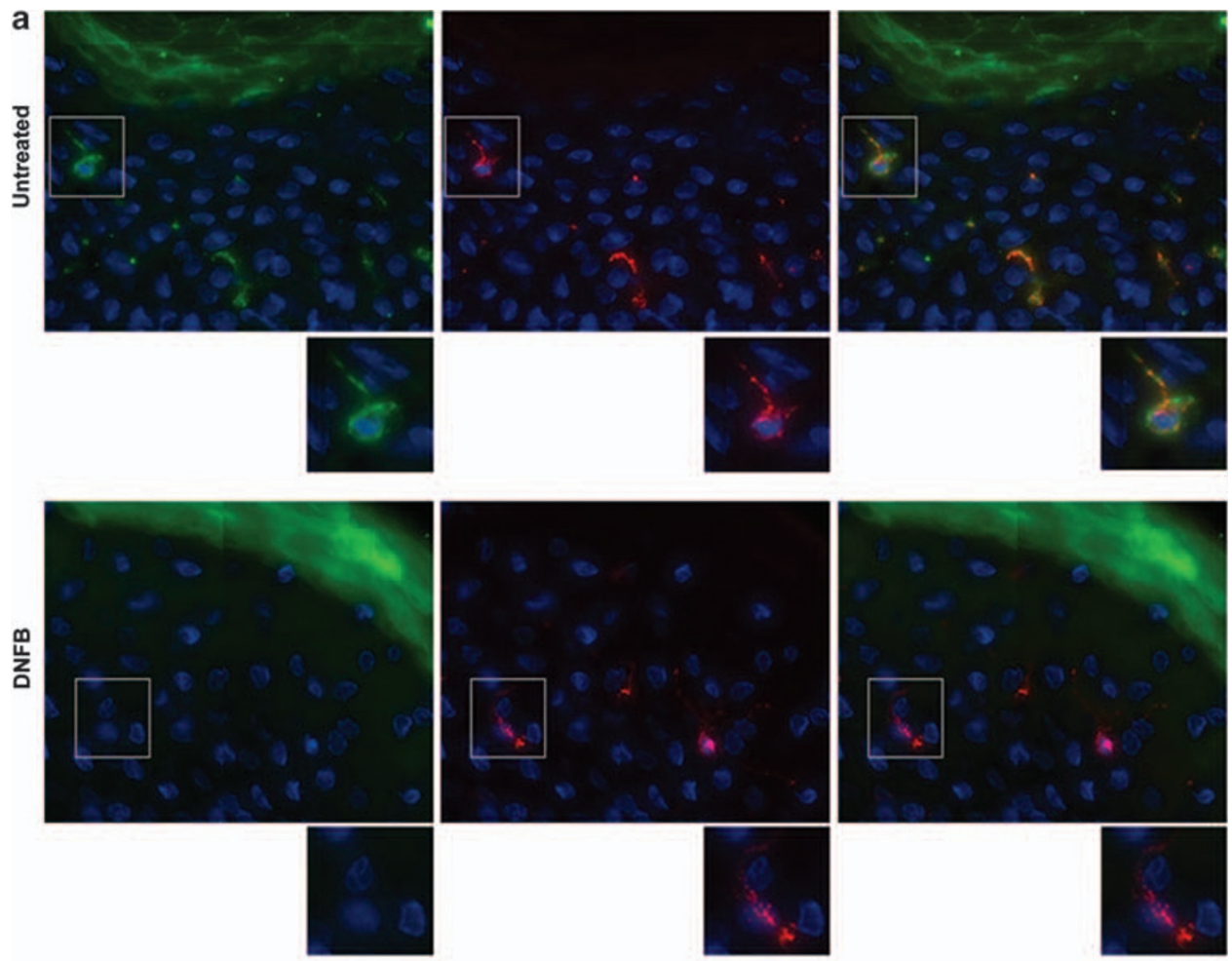

b

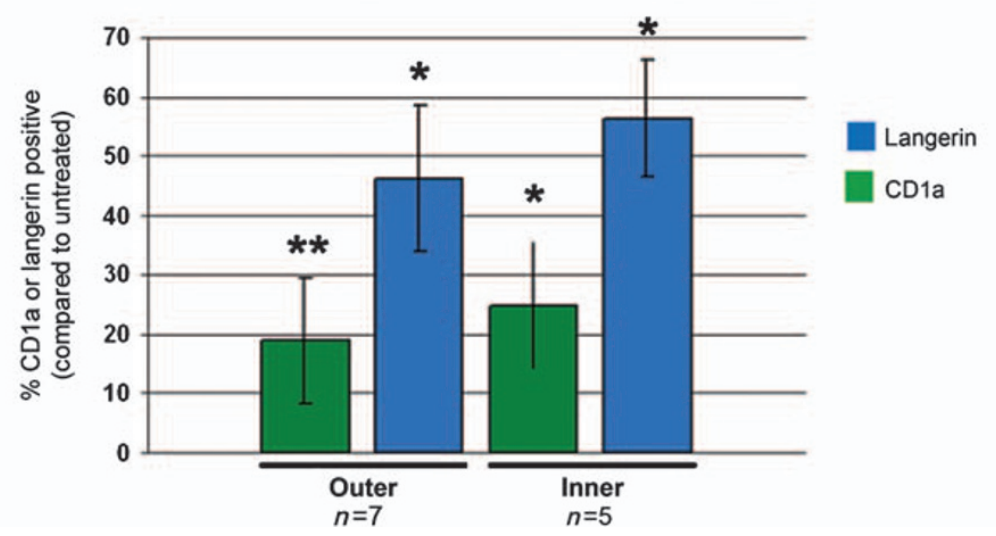

Figure 1 LC marker expression after DNFB exposure. Outer and inner foreskin explants were cultured in media alone or with $0.2 \%$ DNFB for 4-6h. (a) Explants were then frozen, sectioned, and stained for CD1a (green), langerin (red), and DNA (blue). The images show examples of staining in the outer foreskin (see Supplementary Figure 1 online for images of inner foreskin). Insets of selections show enlarged examples of cell marker expression. (b) Average percent of CD1a- or langerin-positive cells normalized to the number of CD1a-positive cells $/ 100 \mu \mathrm{m}^{2}$ of epithelium in untreated cultures. The $n$ values are the number of specimens analyzed. The error bars represent the standard error of the mean. Statistical significance, compared with untreated explants, $\leq 0.05$ is denoted by $\mathrm{a}^{*}$ and significance $<0.02$ is indicated by ${ }^{\star *}$, on the basis of Wilcoxon sign ranked test. DNFB, dinitrofluorbenzene; LC, Langerhans cell.

(top row) where a minimal basal level of CD86 is observed. We did not detect any change in the CD86 expression of LCs in the outer foreskin upon culturing with the cytokine. The area of CD86 fluorescence of several individual cells (total number of cells listed under each bar of graph) was measured for each specimen ( $n$ value). These values were then averaged and CD86 expression in untreated and TNF- $\alpha$-treated explants of outer and inner foreskin were compared. The area of CD86 expression of LCs in the inner foreskin significantly increased 3.4-fold from $12.4 \mu \mathrm{m}^{2} \pm 4.2$ to $42.3 \mu \mathrm{m}^{2} \pm 2.8$ (Figure $4 \mathbf{b}$ ). By contrast, outer foreskin samples showed a small decrease in CD86 expression upon culturing with TNF- $\alpha$. This difference in response suggests that these two areas of the genital epithelium respond differently to cytokine stimulation.

\section{Stimulation of emigration of CD4 + T-cells into the epithelium of the inner foreskin}

Typically, the majority of T-cells in the skin reside below the basement membrane. However, they can infiltrate the epithelium as part of an inflammatory response. Therefore, we were interested to know if there were any changes in epithelial T-cell density upon treatment of the explants. We also 

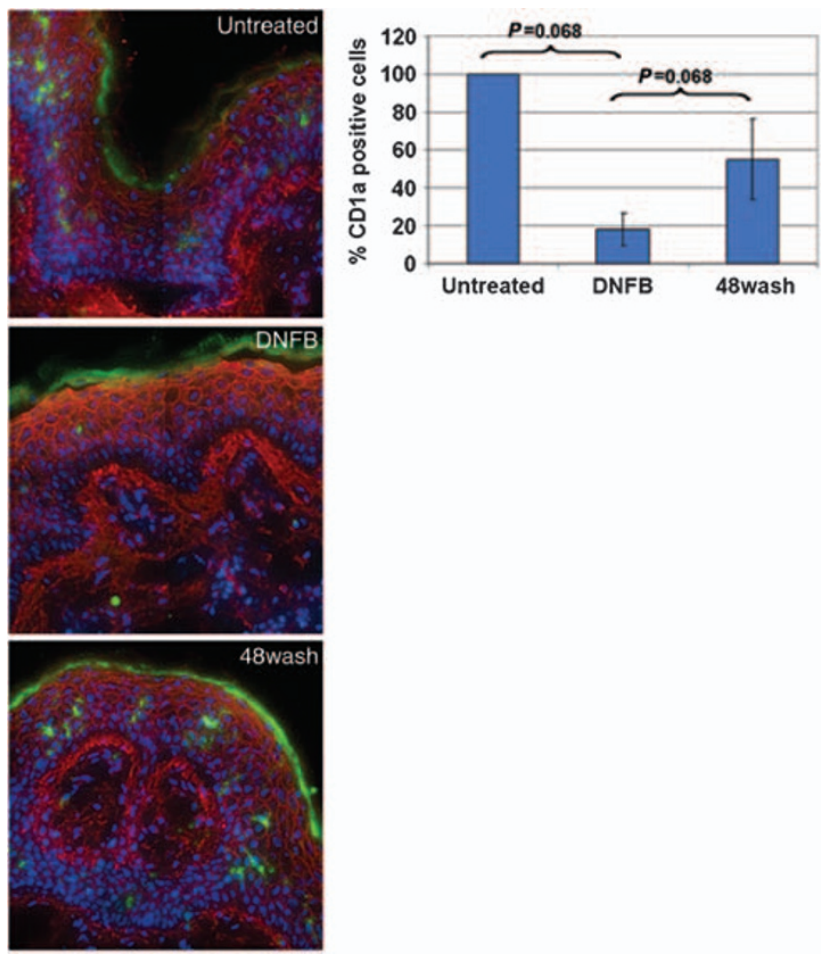

Figure 2 Recovery of LC CD1a expression after DNFB washout. Explants of outer foreskin from four different donors were cultured in media alone or with $0.2 \%$ DNFB. After 3-4 h, the explants were frozen or washed with media and cultured for an additional 2 days before freezing. Sections were stained with WGA (red), CD1a (green), and Hoechst (blue). The number of CD1a-expressing cells and the area of the epithelium were determined. The images show representative views of control, short-term DNFB-treated (DNFB), and DNFB washout (48wash) explants. The average percent of CD1a-positive cells $/ 100 \mu \mathrm{m}^{2}$ of epithelium, normalized to untreated control, over four donor samples was calculated and is depicted in the graph above. The error bars represent the standard error of the mean. The $P$-values shown on graph are calculated on the basis of Wilcoxon sign ranked test. DNFB, dinitrofluorobenzene; LC, Langerhans cell; WGA, wheat-germ agglutinin.

wished to expand our survey of activating compounds to include cytokines that previously had a link to stimulation and migration of CD4 + T-cells. In addition to TNF- $\alpha$, we added treatments with interleukin-12 (IL-12), IL-1 $\beta$, IL-16, CCL21, macrophage inflammatory protein (MIP1 $\alpha$ ), and monocyte chemotactic protein 1 (MCP1). ${ }^{22-31}$ Explants of outer and inner foreskin were left untreated or cultured with one of the above compounds for 2-3 days before freezing and staining. $\mathrm{T}$-cells were identified on the basis of CD4 expression, small size, and morphology. The number of T-cells in each image was determined and the average density of CD4 + T-cells in the epithelium was calculated after each treatment (see Supplementary Table 1 online). As often occurs with donor tissue, there was variability in basal T-cell densities among the samples. Therefore, to determine whether any changes occurred between untreated and treated explants of the entire data set, we calculated the fold increase in density with each treatment, compared with that in untreated outer or inner foreskin. In the charts in Figure 5a, each different colored dot

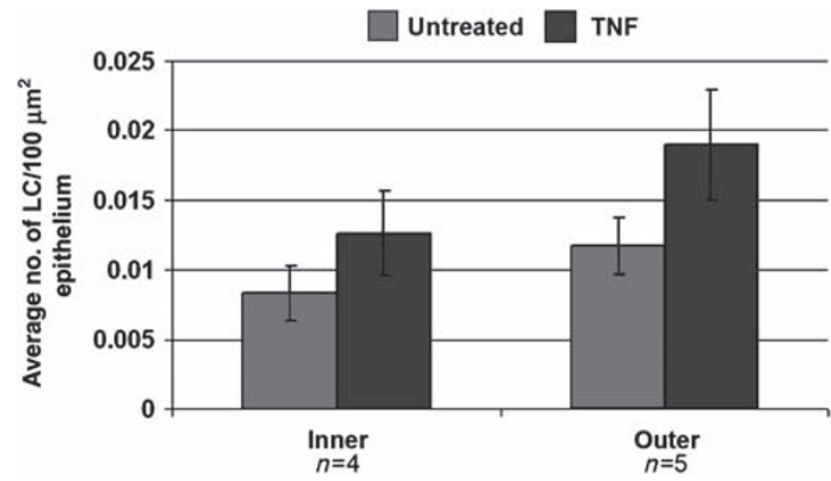

Figure 3 Effect of TNF- $\alpha$ on the number of LCs in adult foreskin. Outer and inner foreskin explants were cultured in media alone or media with TNF- $\alpha$ for 2-3 days. Explants were frozen, sectioned, and stained with WGA, CD1a, and Hoechst. The number of LCs in the epithelium was calculated as described in the text and under section Methods. The graph shows the average density of LCs $/ 100 \mu \mathrm{m}^{2}$ of epithelium. The $n$ values represent the number of donor specimens analyzed. The error bars represent the standard error of the mean. The differences observed were not statistically significant as determined by both Wilcoxon sign ranked test and Student's t-test. LC, Langerhans cell; TNF, tumor necrosis factor; WGA, wheat-germ agglutinin.

represents the fold value of a different donor, normalized to that specimen's untreated control, and the black bar indicates the average of these values. Consistent CD4 T-cell infiltration was observed in treated inner foreskin explants. Specifically, there were statistically significant increases of $2.2 \pm 0.48$ and $2.7 \pm 0.54$-fold in CD4 T-cell density after treatment of inner foreskin with MIP- $1 \alpha$ or TNF- $\alpha$, respectively. Figure $5 \mathbf{b}$ shows an example of an influx of CD4 + T-cells in inner foreskin exposed to MIP-1 $\alpha$. The overall lack of response in the outer foreskin further supports the increased ability of inner foreskin target cells to respond to external stimuli.

After observing increase in T-cell density with MIP-1 $\alpha$, we wished to confirm if its stimulatory effect was specific to T-cells or would also promote LC activation. Therefore, we used the same samples as above and stained for CD86 and CD1a to determine the activation state of the LCs, as described for Figure 4. We did not observe any significant increases in LCs associated CD86 expression with any of the new treatments in either outer or inner foreskin (see Supplementary Figure 2 online). Overall, it appears that migration of T-cells can be affected by more than one stimuli, and that the inner foreskin is more responsive to inflammatory stimuli than outer foreskin.

\section{Environmental sampling by LCs in the inner foreskin}

Images during the above analysis were acquired in explants with an intact epithelial surface (confirmed through WGA staining). LCs resident within both inner and outer foreskin responded to DNFB by downregulating CD1a expression, but stimulation of LCs and T-cells only occurred in the inner foreskin. This suggests that cells of the inner foreskin have better access and/or stronger responses to compounds in the external environment. To test the ability of tissue-resident LCs to gain access to proteins in the environment we cultured explants with a labeled langerin antibody. We hypothesized 


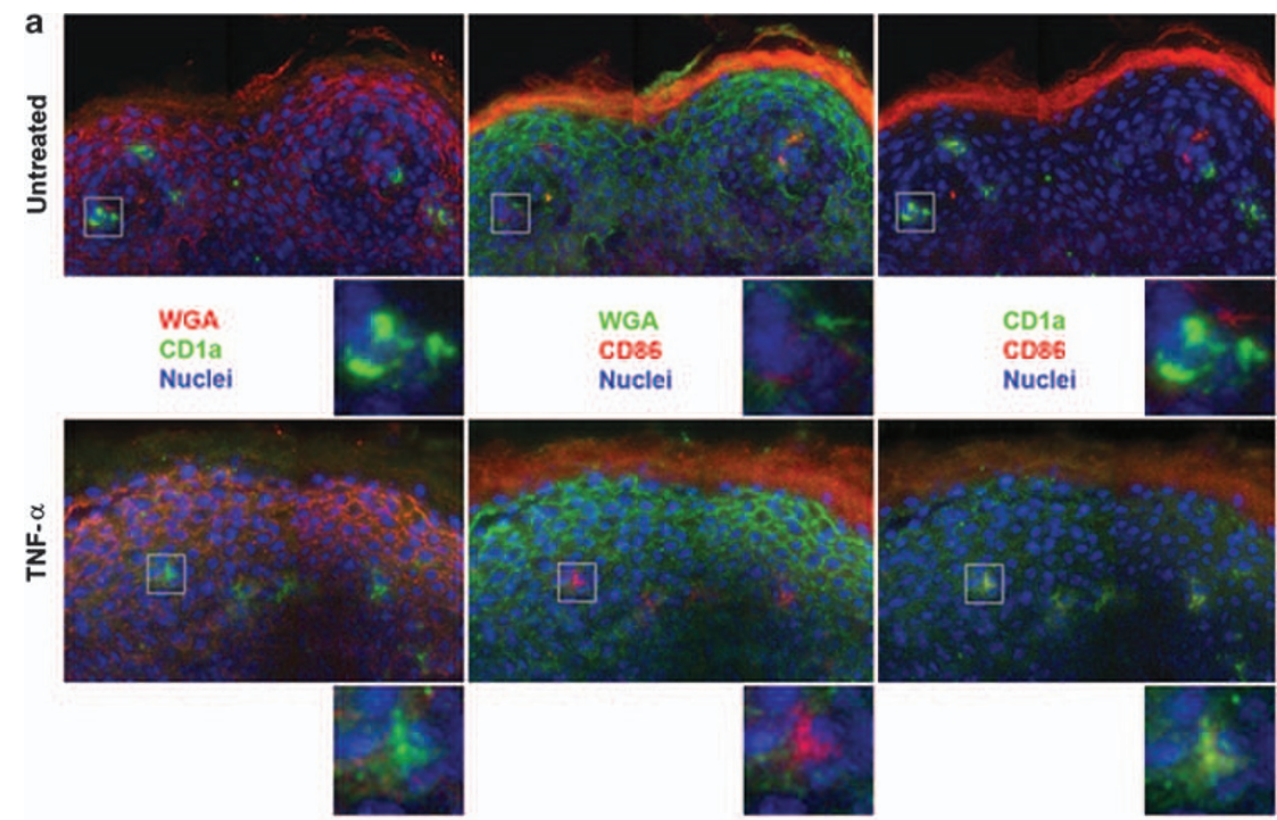

b

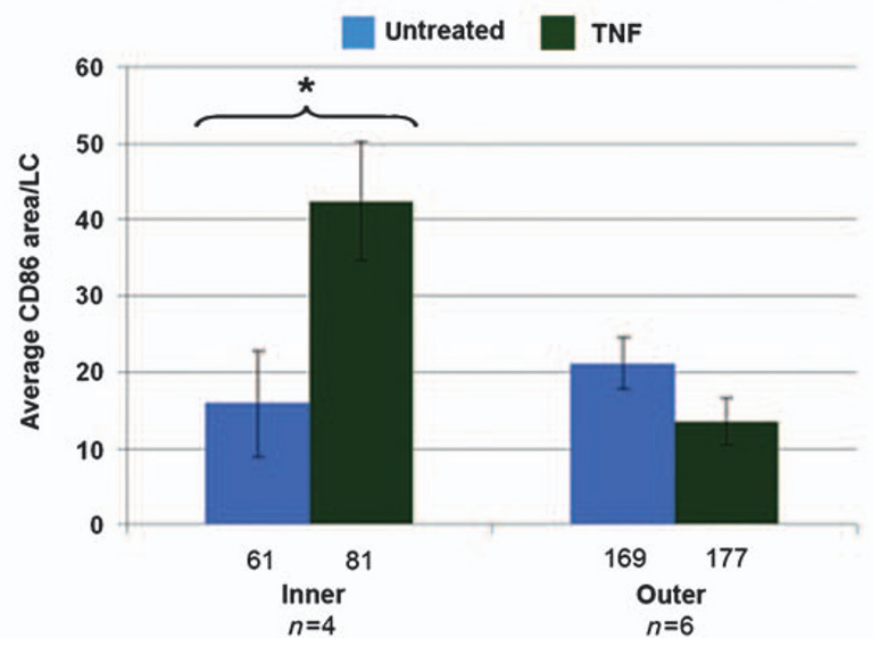

Figure 4 Activation of LCs after exposure to TNF- $\alpha$. Explants of outer and inner foreskin were cultured in media alone or media with TNF- $\alpha$ for $2-3$ days. The explants were frozen, sectioned, and stained with WGA, Hoechst, CD86, and CD1a. (a) Comparison of CD1a-associated CD86 levels in untreated and TNF- $\alpha$-treated inner foreskin. (b) The average area of CD86 fluorescence from several donors. The number of LCs analyzed for each treatment is indicated under the respective bar; the $n$ values represent total number of donor samples processed. Statistical significance $<0.05$ by Wilcoxon sign ranked test is denoted by an *. The error bars represent the standard error of the mean. LC, Langerhans cell; TNF, tumor necrosis factor; WGA, wheat-germ agglutinin.

that LCs of inner foreskin would have better access to the antibody and more robust LC labeling than LCs of the outer foreskin. Langerin antibody was labeled with a green fluorophore and cultured with explants for $4 \mathrm{~h}$ before any freezing or fixation, and is therefore termed 'explant langerin'. Once the explants were frozen and sectioned they were fixed, permeabilized, and stained as usual with a red langerin antibody, referred to as 'section langerin' stain. This second langerin incubation, after freezing, would identify all of the LCs in the tissue section, whereas a yet to be determined number of LCs could be associated with the langerin antibody cultured with the explant. A total of six different donor specimens were treated and the slides were blinded before analysis. An example of the observed staining is shown in Figure 6a. Red section langerin signal is apparent in both outer and inner foreskin; however, explant langerin signal is much more distinct in the inner foreskin (Figure 6a). We compared the number of LCs positive for green langerin signal to the total number of LCs, identified by the red langerin stain, and calculated the percent of LCs that were able to acquire langerin antibody during culture (Figure 6b). Overall only $45 \% \pm 12$ LCs were positive for explant langerin in the outer foreskin. This compares to a significantly higher average of $83 \% \pm 7 \mathrm{LCs}$ positive in the inner foreskin. During analysis we observed that the strength of explant langerin signal varied in the LCs; therefore, we performed additional analyses to include the average intensity of the green signal for each of the LCs. In all six specimens, the average signal of explant langerin in 

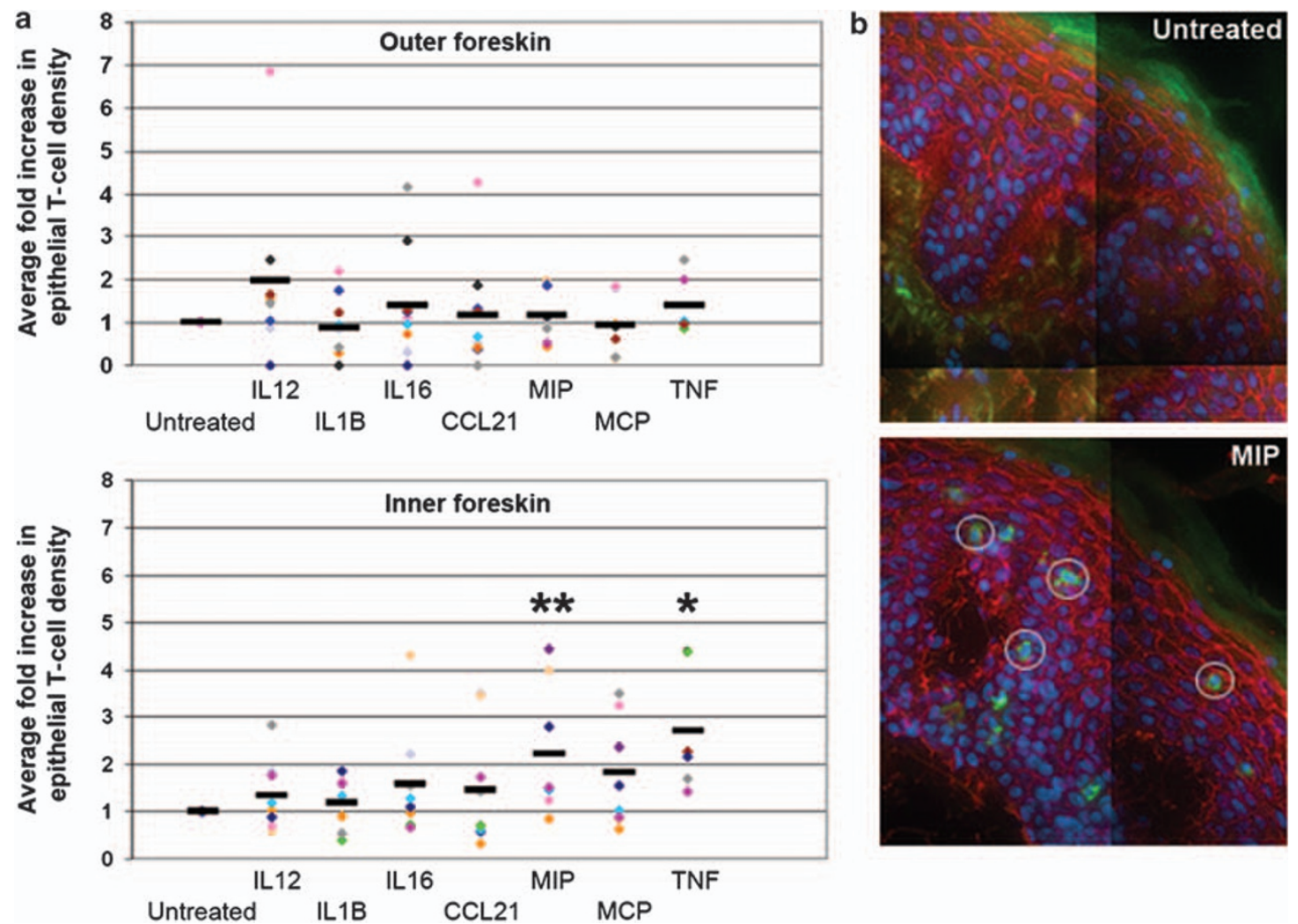

Figure 5 Effect of cytokines on CD4 T-cell concentration in the epithelium. Explants of outer and inner foreskin were cultured in media alone or media with compounds for 2-3 days. The explants were frozen, sectioned, and stained with WGA (red), CD4 (green), and Hoechst (blue). (a) The number of CD4 T-cells was calculated as described in the text and the average fold increase in epithelial CD4 T-cell density/100 $\mu \mathrm{m}^{2}$ of epithelium was calculated from several donors (see Supplementary Table 1 online for actual density values). Statistical significance with a $P$-value $<0.05$ is denoted by an * and significance $<0.02$ is indicated by ${ }^{* \star}$, on the basis of Wilcoxon sign ranked test. Note: Untreated values of zero for two of outer foreskin donors were adjusted to 1 cell/total epithelial area imaged to calculate fold increase. (b) Representative image of untreated and MIP-1 $\alpha$-treated inner foreskin. Some CD4+ T-cells are circled as examples. WGA, wheat-germ agglutinin.

the LCs of the inner foreskin was brighter than that in the outer foreskin (Figure $\mathbf{6 c}$ ). When averaged, the LCs of the inner foreskin had a twofold higher intensity than those in outer foreskin (readings of $338 \pm 93$ vs. $153 \pm 66$, respectively). Finally, we also measured the area of green, explant langerin, signal for each LC and calculated the relative amount of antibody retrieved by each cell (Figure 6d). The average area was 3.2-fold higher in the inner foreskin $\left(48 \mu \mathrm{m}^{2} \pm 14\right)$ than in the outer foreskin $\left(15 \mu \mathrm{m}^{2} \pm 6\right)$. These data clearly suggest that intact inner foreskin has greater access to materials in its external environment than the outer foreskin.

\section{DISCUSSION}

Several clinical trials have shown a link between circumcision and decrease in HIV acquisition. ${ }^{9-13}$ However, the mechanism through which foreskin removal has a protective effect is unknown. Of related significance is the increased incidence of HIV infection during co-infection with other sexually transmitted agents. ${ }^{4-8}$ The experiments here potentially link these two factors and propose a cause-and-effect relationship of inflammation of the inner foreskin and activation/proximity change of immune cells significant to HIV transmission.
Our previous report of sustained viability and structure of cultured foreskin explants ${ }^{14}$ did not consider the physiological state of resident HIV target cells. Here we observe the response of resident LCs and T-cells to external stimuli after days in culture, either by changing their protein expression or location within the epithelium. In the experiments with DNFB we observed that the LCs specifically downregulated their surface marker CD1a, but were able to renew its expression upon DNFB removal (Figures 1 and 2). These responses to a compound in the culture media prompted us to analyze the immune cells of the outer and inner foreskin, specifically to compare the responses of the LCs and CD4+ T-cells to inflammatory cytokines.

We used upregulation of the activation marker CD86 and infiltration of T-cells into the epithelium as hallmarks of an inflammatory response in the tissue and observed a clear disparity between the observations in the outer and inner foreskin. Measurable changes, such as activation of LCs and infiltration of CD4 + T-cells into the epithelium, were only observed in the inner foreskin (Figures 4 and 5). Specifically, TNF- $\alpha$ significantly promoted both responses, whereas MIP$1 \alpha$ induced significant increases only in epithelial CD $4+$ T-cell density. 
a
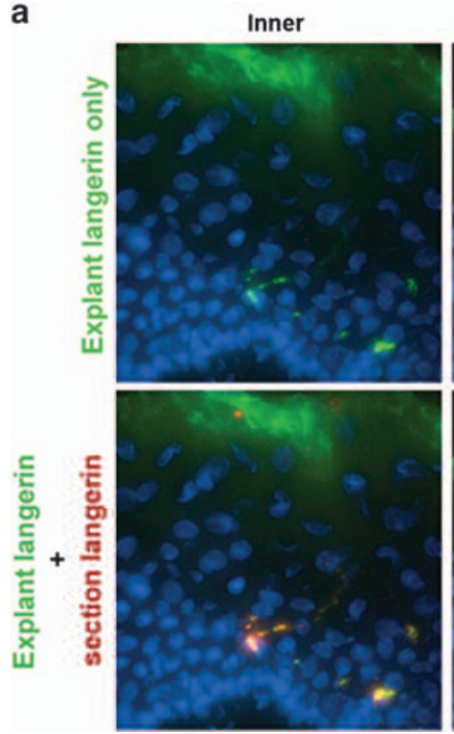
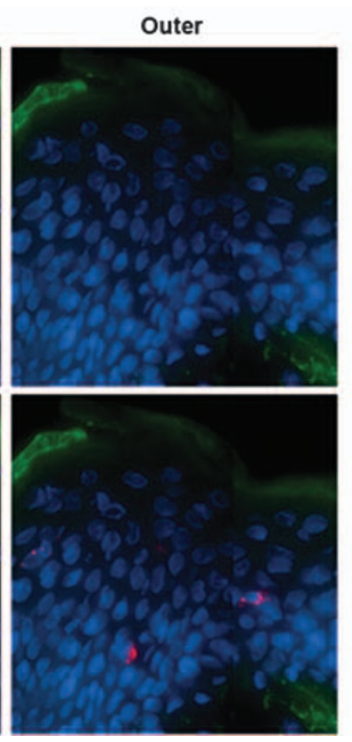

b
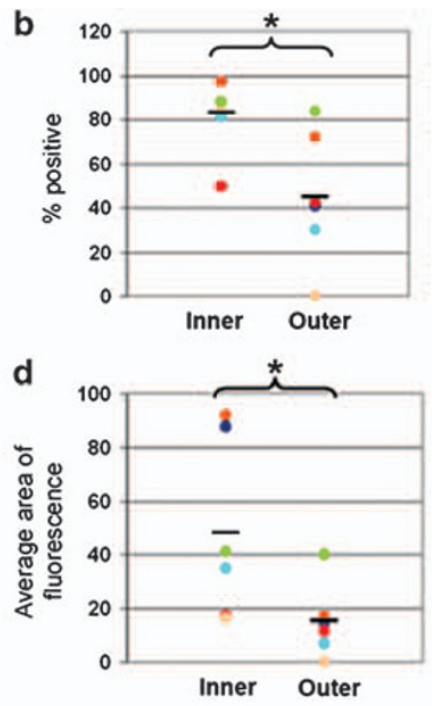

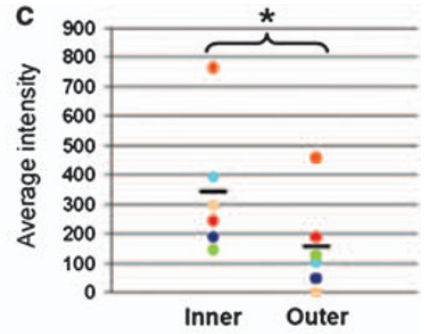

Figure 6 Characterization of fluorescence-labeled antibody acquired by tissue-resident LCs. Explants of outer and inner foreskin were incubated with green fluorescent langerin (explant langerin) while in culture, before freezing. (a) The frozen explants were then sectioned and stained with red langerin antibody (section langerin) and Hoechst (blue). The bottom row shows all staining, and the top row has the red signal removed to show green signal within the LCs of the inner foreskin. Several images of each donor tissue were taken to measure and calculate: (b) the percent of LCs labeled with explant langerin, (c) the average intensity of explant langerin signal per cell, and (d) the average area of explant langerin signal per cell. Each different colored dot represents the average value for each donor specimen; the black bar is the average of all donors. Statistical significance $<0.05$, calculated by Wilcoxon sign ranked test, is denoted by an *. LC, Langerhans cell.

The selective response of HIV target cells residing in the inner foreskin suggests some physiological difference between the outer and inner foreskin. Either the resident cells of the inner foreskin are more responsive to external stimuli or the compounds added are better able to penetrate its epithelial barrier. Both aspects of the foreskin responded similarly to DNFB (Figure 1); however, this is a small molecule that we believe can likely penetrate both inner and outer foreskin tissue with similar efficiency. By contrast, we observed cellular responses in the inner, but not outer, foreskin when explants were exposed to larger proteins like TNF- $\alpha$. In addition, a greater number of LCs resident in the inner foreskin were able to access the external antibody in culture (Figure 6b) and were also able to 'collect' the antibody to a greater degree than those in the outer foreskin (Figure $6 \mathrm{c}$ and $\mathbf{d}$ ).

These observations suggest a new model of how HIV transmission may be facilitated by the presence of foreskin. The foreskin forms a mucosal surface that more efficiently detects environmental factors, increasing inflammatory responses. Consistent with the known ability of inflammation to increase HIV acquisition, the increased inflammatory response associated with the inner foreskin would lead to a higher efficiency of transmission, as seen with HSV-2 infection. ${ }^{32}$ Interestingly, this model is consistent with the increased acquisition of HIV by uncircumcised individuals who received the Ad5 HIV vaccine used in the STEP vaccine trial. ${ }^{11}$ These individuals had a greater than twofold increase in HIV acquisition when compared with circumcised individuals who received the vaccine. We theorize that presence of inner foreskin in vaccinated individuals led to detection of HIV antigens through the inner foreskin and a subsequent antigen-specific inflammatory response. The resulting activation and infiltration of target cells would increase the likelihood of transmission. By contrast, the more responsive HIV target cells in the inner foreskin were not present in the circumcised individuals. Therefore, they had less of an inflammatory response to HIV antigens and no increase in the efficiency of HIV acquisition.

This model for how circumcision might decrease HIV acquisition has important implications for strategies to reduce the transmission of HIV. For example, treatments that decrease the permeability of the inner foreskin would decrease inflammatory responses and subsequent activation and infiltration of HIV target cells. Other approaches could involve blunting the immune response. As recently suggested by Hasse and co-workers, ${ }^{33}$ the antimicrobial and anti-inflammatory compound glycerol monolaurate could prevent the vaginal transmission of SIV. Microbicides designed to block the inner foreskin responses described here could represent a novel approach for prevention of HIV acquisition.

\section{METHODS}

Tissue source. Foreskin tissue was obtained from adult male patients undergoing elective circumcisions at local hospitals. All tissue was obtained according to institutional guidelines. Donor tissue was obtained the same day as the surgery was performed and kept in sterile saline solution until processing, within $24 \mathrm{~h}$ of surgery.

Tissue culture and cytokine treatments. The outer and inner foreskin was cut separate from each other upon receipt of the specimen. Explants of approximately $1 \mathrm{~cm}^{2}$ of each were cut and kept in sterile 
phosphate-buffered saline during the course of tissue processing. A 48-well plate was prepared with wells containing RPMI with $10 \%$ fetal bovine serum alone for untreated samples, or had media containing IL-12 (100 $\left.\mathrm{ng} \mathrm{ml}^{-1}\right)$, IL-16 (500 $\left.\mathrm{ng} \mathrm{ml}^{-1}\right)$, CCL21 (100 $\left.\mathrm{n} \mathrm{ml}^{-1}\right)$, IL$1 \mathrm{~B}\left(100 \mathrm{ng} \mathrm{ml}^{-1}\right)$, MIP-1 $\alpha\left(100 \mathrm{ng} \mathrm{ml}^{-1}\right)$, monocyte chemoattractant protein-1 $\left(100 \mathrm{ng} \mathrm{ml}^{-1}\right)$, or TNF- $\alpha\left(30 \mathrm{ng} \mathrm{ml}^{-1}\right)$ (Peprotech, Rocky Hill, NJ). The explants were kept in culture for 2-3 days and then were frozen in OCT before sectioning and staining.

\section{DNFB experiments}

Explants were prepared as described above. The wells of a 48 -well plate were filled with either RPMI with $10 \%$ fetal bovine serum alone or with $0.2 \%$ DNFB (Sigma, St Louis, MO). After culturing for the desired time period (typically $4 \mathrm{~h}$ ), the explants were frozen in OCT before sectioning and staining. For washout experiments, untreated and DNFB-treated explants were frozen after 3-4 $\mathrm{h}$ and an additional treated explant was washed with media several times and then cultured for an additional 2 days before being frozen. The epithelial area was measured and the number of CD1a-positive cells was counted. The number of CD1a- or langerin-positive cells/ $100 \mu \mathrm{m}^{2}$ of untreated tissue was used as a reading of $100 \%$ for normalization of DNFBtreated and washout tissue.

Immunofluorescence and antibodies. Sections were fixed in methanol: acetone (1:1) and blocked with normal donkey serum before staining. To, identify LCs, sections were stained with CD1a (OKT6 hybridoma; ATCC, Manassas, VA). Activation status was identified by staining with CD1a in conjunction with CD86 (BD Pharmingen, San Diego, CA) labeled with Zenon Alexafluor-594 (Invitrogen, Eugene, OR) Sections were also stained with WGA and Hoechst. To identify CD4+ T-cells, additional sections were stained with CD1a, Alexafluor-594 (Invitrogen)-labeled CD4 monoclonal antibody (Sigma), WGA, and 4',6-diamidino-2-phenylindole. For co-staining of langerin and CD1a for DNFB experiments, OKT6 followed by anti-mouse Oregon Green secondary (Invitrogen) and Alexafluor-594 (Invitrogen)-labeled langerin (Beckman Coulter, Brea, CA) were used. After staining, sections were covered with mounting medium (Dako, Glostrup, Denmark) and coverslips, and sealed with nail polish until imaged.

Antibody uptake experiments. Explants of outer and inner foreskin were cultured in media containing langerin antibody labeled with Zenon Alexafluor- 488 for $4 \mathrm{~h}$. The explants were frozen in OCT, sectioned, fixed with $3.7 \%$ paraformaldehyde, and stained with langerin labeled with Zenon Alexafluor-594, WGA, and Hoechst. Slides were blinded before imaging and analysis. One donor specimen was excluded due to excessive inflammation of the tissue that would potentially perturb results. Half of these samples (with sections from deeper within the explant) were analyzed in duplicate for antibody uptake to confirm reproducibility of results. These data were not represented in the graphs and included in the data presented here, but did show a similar trend of more antibody-positive cells in the inner foreskin than in the outer foreskin.

Image acquisition and analysis. All images were acquired using an Olympus Deltavision IX71 deconvolution microscope. Panel images were acquired so that overall structure of the surface epithelium in addition to the underlying connective tissue could be observed. The total number of images included a minimum of $1 \mathrm{E} 5 \mu \mathrm{m}^{2}$ of epithelial area for each treatment. Images for each individual experiment were acquired with the same settings (exposure time, exposure strength, and number of $z$-sections) and on the same day for untreated and treated samples to assure consistency in detecting fluorescence intensity. Samples were blinded either before imaging or analysis. The Softworx 3.5.1 analysis software was used to measure the area of epithelium to determine cell densities within the tissue. Polygon modeling functions of the software allowed us to measure the area of fluorescence of specified fluors for LC activation and antibody uptake studies. The mean fluorescence intensity of the LC polygon was also recorded for the antibody uptake experiments.

Statistical analysis. Measurements from several images of each treatment were used to calculate an average value for a specific treatment for each assay. Error bars and \pm notations signify the standard error of the mean. Statistical significance analysis was performed using an SPSS program and by Wilcoxon sign ranked sum test. The $P$-values are listed in each respective figure legend. Data that were deemed statistically significant by the Wilcoxon test were also found to be significant by Student's $t$-test.

SUPPLEMENTARY MATERIAL is linked to the online version of the paper at http://www.nature.com/mi

\section{ACKNOWLEDGMENTS}

We thank the Urology department and the Pathology laboratory of Northwestern Memorial Hospital and Rush University Medical Center of Chicago, Illinois, and the patients for their cooperation in tissue specimen collection. This work was supported by NIH grants AI052051 and Al076968.

AUTHOR CONTRIBUTIONS: K.M.F. and S.M.B. contributed equally to the experiments; M.R.A. helped with imaging and analysis; K.M.F. and S.M.B. designed, performed, and analyzed the experiments; K.M.F. compiled the data and wrote the paper; S.M.B. assisted with paper editing; and T.J.H. reviewed and discussed the experiments and assisted with manuscript editing.

() 2010 Society for Mucosal Immunology

\section{REFERENCES}

1. Cunningham, A.L., Carbone, F. \& Geijtenbeek, T.B. Langerhans cells and viral immunity. Eur. J. Immunol. 38, 2377-2385 (2008).

2. de Jong, M.A. \& Geijtenbeek, T.B. Human immunodeficiency virus-1 acquisition in genital mucosa: Langerhans cells as key-players. J. Intern. Med. 265, 18-28 (2009).

3. Chieppa, M., Rescigno, M., Huang, A.Y.C. \& Germain, R.N. Dynamic imaging of dendritic cell extension into the small bowel lumen in response to epithelial cell TLR engagement. J. Exp. Med. 203, 2841-2852 (2006).

4. Abu-Raddad, L.J. et al. Genital herpes has played a more important role than any other sexually transmitted infection in driving HIV prevalence in Africa. PLoS One 3, e2230 (2008).

5. Benki, S., McClelland, R.S. \& Overbaugh, J. Risk factors for human immunodeficiency virus type-1 acquisition in women in Africa. J. Neurovirol. 11 (Suppl 1), 58-65 (2005).

6. Galvin, S.R. \& Cohen, M.S. The role of sexually transmitted diseases in HIV transmission. Nat. Rev. Microbiol. 2, 33-42 (2004).

7. Haaland, R.E. et al. Inflammatory genital infections mitigate a severe genetic bottleneck in heterosexual transmission of subtype $\mathrm{A}$ and $\mathrm{C}$ HIV-1. PLoS Pathog. 5, e1000274 (2009).

8. Sagar, M. et al. Identification of modifiable factors that affect the genetic diversity of the transmitted HIV-1 population. Aids 18, 615-619 (2004).

9. Auvert, B. et al. Randomized, controlled intervention trial of male circumcision for reduction of HIV infection risk: the ANRS 1265 trial. PLOS Med. 2, e298 (2005)

10. Bailey, R.C. et al. Male circumcision for HIV prevention in young men in Kisumu, Kenya: a randomised controlled trial. Lancet 369, 643-656 (2007).

11. Buchbinder, S.P. et al. Efficacy assessment of a cell-mediated immunity HIV-1 vaccine (the Step Study): a double-blind, randomised, placebocontrolled, test-of-concept trial. Lancet 372, 1881-1893 (2008).

12. Gray, R.H. et al. Male circumcision for HIV prevention in men in Rakai, Uganda: a randomised trial. Lancet 369, 657-666 (2007).

13. Sullivan, P.S. et al. Male circumcision for prevention of HIV transmission: what the new data mean for HIV prevention in the United States. PLOS Med. 4, e223 (2007).

14. Fischetti, L., Barry, S.M., Hope, T.J. \& Shattock, R.J. HIV-1 infection of human penile explant tissue and protection by candidate microbicides. Aids 23, 319-328 (2009). 


\section{ARTICLES}

15. Mohr, R.E. \& Takashima, A. Epidermal Langerhans cell movement in situ: a model for understanding immunologic function in the skin. Arch. Dermatol. 143, 1352 (2007).

16. Pistoor, F.H. et al. Novel predictive assay for contact allergens using human skin explant cultures. Am. J. Pathol. 149, 337-343 (1996).

17. Teunissen, M.B. Dynamic nature and function of epidermal Langerhans cells in vivo and in vitro: a review, with emphasis on human Langerhans cells. Histochem. J. 24, 697-716 (1992).

18. Valladeau, J., Dezutter-Dambuyant, C. \& Saeland, S. Langerin/CD207 sheds light on formation of Birbeck granules and their possible function in Langerhans cells. Immunol. Res. 28, 93-107 (2003).

19. de Jong, M.A. et al. TNF-alpha and TLR agonists increase susceptibility to HIV-1 transmission by human Langerhans cells ex vivo. J. Clin. Invest. 118, 3440-3452 (2008).

20. Fahrbach, K.M. et al. Activated CD34-derived Langerhans cells mediate transinfection with human immunodeficiency virus. J. Virol. 81, 6858-6868 (2007)

21. Caux, C. et al. Activation of human dendritic cells through CD40 crosslinking. J. Exp. Med. 180, 1263-1272 (1994).

22. Baggiolini, M. \& Loetscher, P. Chemokines in inflammation and immunity. Immunol. Today 21, 418-420 (2000).

23. Carr, M.W., Roth, S.J., Luther, E., Rose, S.S. \& Springer, T.A. Monocyte chemoattractant protein 1 acts as a T-lymphocyte chemoattractant. Proc. Natl. Acad. Sci. USA 91, 3652-3656 (1994).

24. Cumberbatch, M., Dearman, R.J. \& Kimber, I. Interleukin 1 beta and the stimulation of Langerhans cell migration: comparisons with tumour necrosis factor alpha. Arch. Dermatol. Res. 289, 277-284 (1997).

25. Enk, A.H., Angeloni, V.L., Udey, M.C. \& Katz, S.I. An essential role for Langerhans cell-derived IL-1 beta in the initiation of primary immune responses in skin. J. Immunol. 150, 3698-3704 (1993).

26. Jakob, T., Ring, J. \& Udey, M.C. Multistep navigation of Langerhans/ dendritic cells in and out of the skin. J. Allergy Clin. Immunol. 108, 688-696 (2001).

27. Koch, S., Kohl, K., Klein, E., von Bubnoff, D. \& Bieber, T. Skin homing of Langerhans cell precursors: adhesion, chemotaxis, and migration. J. Allergy Clin. Immunol. 117, 163-168 (2006).

28. Loetscher, P., Seitz, M., Clark-Lewis, I., Baggiolini, M. \& Moser, B. Monocyte chemotactic proteins MCP-1, MCP-2, and MCP-3 are major attractants for human CD4+ and CD8+ T lymphocytes. FASEB J. 8, 1055-1060 (1994).

29. Lynch, E.A., Heijens, C.A., Horst, N.F., Center, D.M. \& Cruikshank, W.W. Cutting edge: IL-16/CD4 preferentially induces Th1 cell migration: requirement of CCR5. J. Immunol. 171, 4965-4968 (2003).

30. Rollins, B.J. Chemokines. Blood 90, 909-928 (1997).

31. Zhang, X. et al. Interleukin 12 induces T-cell recruitment into the atherosclerotic plaque. Circ. Res. 98, 524-531 (2006).

32. Brian, R.L. et al. Increased number and function of natural killer cells in human immunodeficiency virus 1 -positive subjects co-infected with herpes simplex virus 2. Immunology 129, 186-196 (2010).

33. Li, Q. et al. Glycerol monolaurate prevents mucosal SIV transmission. Nature 458, 1034-1038 (2009). 